\title{
Multiple valve endocarditis: a Hemi-Commando procedure
}

\author{
Jan Vojacek ${ }^{1}$, Pavel Zacek ${ }^{1}$, Jiri Ondrasek ${ }^{2}$ \\ ${ }^{1}$ Department of Cardiac Surgery, Faculty of Medicine and University Hospital in Hradec Kralove, Hradec Kralove, Charles University, Czech \\ Republic; ${ }^{2}$ Centre of Cardiovascular and Transplantation Surgery, Brno, Czech Republic \\ Correspondence to: Prof. Jan Vojacek, MD, PhD. Department of Cardiac Surgery, University Hospital Hradec Kralove, Sokolska 581, 500 05, Hradec \\ Kralove, Czech Republic. Email: vojacek.jan@fnhk.cz.
}

Submitted May 13, 2019. Accepted for publication Jul 15, 2019.

doi: 10.21037/acs.2019.07.07

View this article at: http://dx.doi.org/10.21037/acs.2019.07.07

\section{Introduction}

Invasive double valve endocarditis (aortic and mitral) involving the fibrous skeleton of the heart requires complex high-risk surgical management. The procedure which was originally suggested by Tirone David, consists of double valve replacement with pericardial or Dacron patches to re-create "neoaortomitral continuity" (1). This operation was named by the Cleveland Clinic group a "Commando procedure", the Leipzig group use the term "UFO procedure" (2). In patients in whom the posterior mitral leaflet is not affected by the infection and at least the free edge of anterior mitral could be spared, a modification of the Commando procedure was suggested and named the "Hemi-Commando procedure" (3).

\section{Clinical vignette}

The following video case report presents the surgical technique of Hemi-Commando procedure. A 62-year-old male patient after a successful cardiopulmonary resuscitation due to onset of ventricular fibrillation was diagnosed with severe aortic stenosis (bicuspid aortic valve, indexed aortic valve area $0.38 \mathrm{~cm}^{2} / \mathrm{m}^{2}$ ) and severe impairment of left ventricular ejection fraction (LVEF) (LVEF $=20 \%)$. Once stabilized, the patient underwent an aortic valve replacement with a bioprosthesis (Mitroflow 25). The postoperative course was uneventful and was finalized by implantable cardioverter defibrillator (ICD) implantation on a basis of poor LVEF and the history of ventricular fibrillation. The patient was discharged from the hospital on postoperative day 12 . Initially he did well but one month later he developed progressive weakness, sweating, and fever. There was an elevation of C-reactive protein
(CRP) and blood cultures were positive for Streptococcus galloticus and coagulase-negative Staphylococcus. A suspicion of prosthetic valve endocarditis (PVE) was confirmed by transoesophageal echocardiography (TEE) which demonstrated vegetations attached to the bioprosthesis, progressive abscess formation with destruction of the intervalvular fibrous body and an anterior mitral valve leaflet (AML) perforation resulting in severe mitral valve regurgitation (Figures 1,2). The patient was septic with signs of malnutrition and congestive heart failure. Aggressive antibiotic treatment was initiated and the patient was indicated for high-risk reoperation (salvage procedure) for double valve endocarditis involving the intervalvular fibrous body. For such infective lesions, the procedure of choice at our institution is the Hemi-Commando procedure with use of aortomitral homograft. At surgery, the HemiCommando procedure was completed but the procedure required long cross-clamping and cardiopulmonary bypass (CPB) times (185 and 250 minutes, respectively). The postoperative course was complicated by multi-organ failure requiring dialysis. The patient was extubated on the third postoperative day. Following a long period of antibiotic treatment and slow rehabilitation he was discharged from the hospital 2.5 months later.

\section{Surgical techniques of Hemi-Commando procedure}

\section{Preparation and exposition}

The operation is performed via median sternotomy. In reoperation, we frequently use peripheral cannulation for $\mathrm{CPB}$. Bicaval venous cannulation is mandatory for extensive exposure with wide opening of the left atrium, and (in 


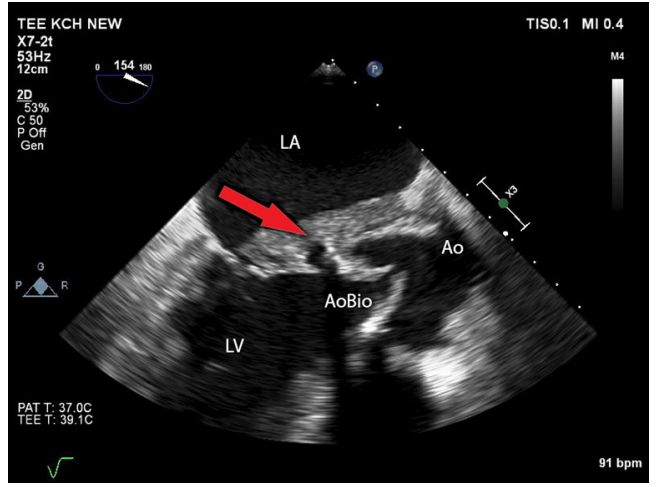

Figure 1 TEE (long axis view)—double valve endocarditis with involvement of aortomitral continuity and the anterior leaflet of the mitral valve. arrow: perforation of the anterior mitral leaflet. TEE, transesophageal echocardiography; AoBio, aortic bioprosthesis; LA, left atrium; LV, left ventricle; Ao, aorta.

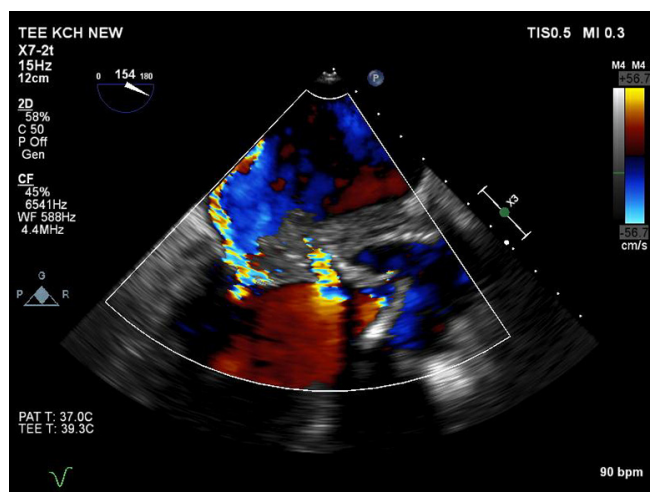

Figure 2 TEE (long axis view, color Doppler). TEE, transesophageal echocardiography.

case of transseptal approach) also of the right atrium. For this reason, in reoperations, we use the neck cannula (venous cannula inserted percutaneously through the jugular vein into the superior vena cava). This approach, together with peripheral cannulation from the groin, facilitates the procedure. These patients are often septic, hemodynamically compromised and the procedure is extensive, with an expected long cross-clamp time. For cardioplegia we use CUSTODIOL ${ }^{\circledR}$ HTK Solution which is delivered in antegrade or antegrade/retrograde fashion.

\section{Operation}

After initiation of CPB and heart arrest, the aorta is

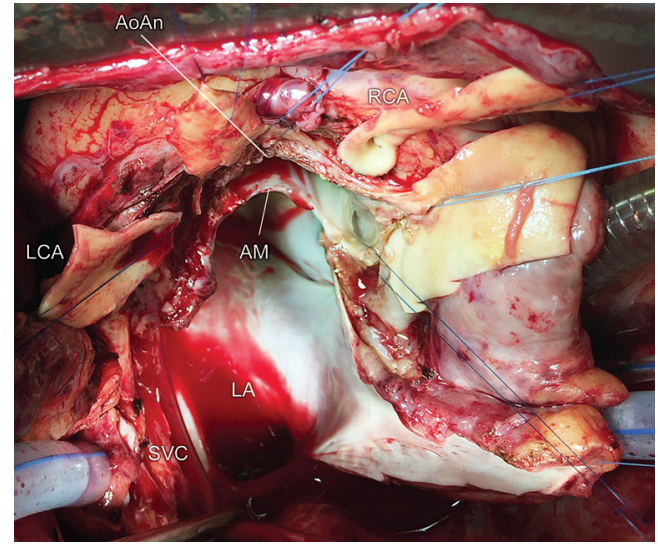

Figure 3 Hemi-Commando procedure-the operative view after removing of all infected aortic root tissue together with aortomitral continuity, and basal portion of the anterior mitral leaflet. AoAn, aortic annulus; LCA, left coronary artery; RCA, right coronary artery; AM, anterior mitral leaflet remnants; LA, left atrium; SVC, superior vena cava.

transected above the sinotubular junction (STJ) and aortic root and the aortic valve is inspected. The infected aortic valve is removed and both coronary buttons are dissected free. In this particular case, the extent of multiple abscess formation was excessive and devastated the aorto-mitral curtain and two thirds of the anterior mitral leaflet. All infected tissue must be radically resected including the annular abscesses, intervalvular fibrous body, and surrounding tissue of the aortic wall and the roof of the left atrium. Opening of the roof of the left atrium is mandatory and we use a biatrial transseptal incision extended into the roof of the left atrium. This approach offers an excellent exposure of the mitral valve and facilitates the radical removal of all infected tissue. This results in a large tissue defect between the aortic root, left ventricular outflow tract, and left atrium with the remnants of AML (its free edge with chordae tendineae) (Figure 3). Suture mitral annuloplasty or implantation of the open band could be performed at this stage. Integrity of the heart is than restored with trimmed incorporated aortomitral homograft. In this aortic homograft, the donor's AML is preserved as well as the rim of the left atrial roof (Figure 4). The homograft is implanted with multiple interrupted nonpledgeted sutures (from anterior to posterior trigone). Before the homograft is seated to its position and the sutures tied, the AML of the homograft is sutured to the free edge of the patient AML using 5/0 monofilament 


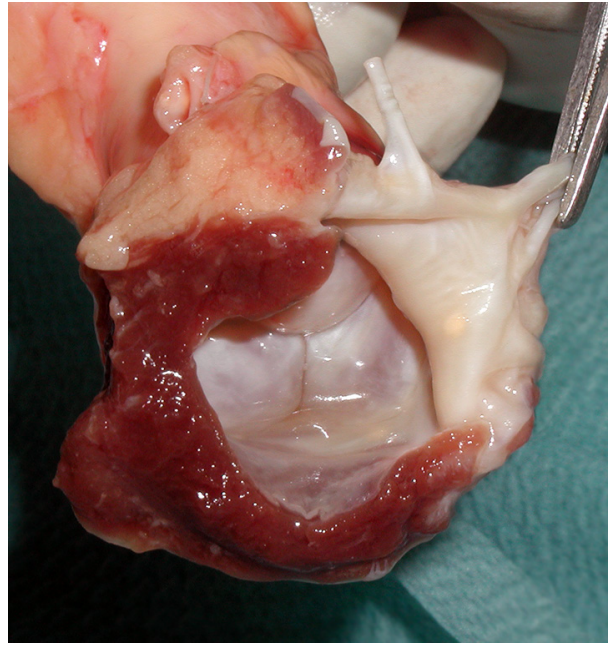

Figure 4 Aortic homograft with preserved anterior leaflet of the mitral valve.

running suture. The roof of the left atrium is closed using the left atrial roof origin on the homograft. The coronary buttons are re-implanted, the homograft is anastomosed to the ascending aorta, and the right atriotomy closed.

\section{Comments}

The advantage of Hemi-Commando over the Commando procedure is the preservation of a substantial part of the native mitral valve. Implantation of incorporated aortomitral homograft restores the unrepeatable beauty of aorto-mitral continuity. This procedure is indicated in patients with root pathology requiring root replacement (e.g., aortic abscess and/or communication to another heart chamber), especially in concomitant involvement of the intervalvular fibrous body and AML. The intact posterior mitral leaflet and free edge of AML are, however, prerequisites for such a technique.

This procedure is performed rarely and long-term data are not available. The only publication dealing with mid- term results involves 37 patients operated on between 2010 and 2017 in Cleveland Clinic (4). In-hospital mortality was $8 \%$ and 1 - and 3 -year survival was $91 \%$ and $82 \%$, respectively (only one death was due to recurrent IE). The Hemi-Commando procedure (similarly to the Commando procedure) is a complex cardiac operation and requires dedicated cardiac surgeons. In selected patients with double valve endocarditis involving the intervalvular fibrous body these operations represent the only surgical solution which can restore the integrity of the heart.

\section{Acknowledgments}

This work was supported by the research program PROGRES Q40-04.

\section{Footnote}

Conflicts of Interest: The authors have no conflicts of interest to declare.

\section{References}

1. David TE, Kuo J, Armstrong S. Aortic and mitral valve replacement with reconstruction of the intervalvular fibrous body. J Thorac Cardiovasc Surg 1997;114:766-71; discussion 771-2.

2. Davierwala PM, Binner C, Subramanian S, et al. Double valve replacement and reconstruction of the intervalvular fibrous body in patients with active infective endocarditis. Eur J Cardiothorac Surg 2014;45:146-52.

3. Navia JL, Al-Ruzzeh S, Gordon S, et al. The incorporated aortomitral homograft: a new surgical option for double valve endocarditis. J Thorac Cardiovasc Surg 2010;139:1077-81.

4. Elgharably H, Hakim AH, Unai S, et al. The incorporated aortomitral homograft for double-valve endocarditis: the 'hemi-Commando' procedure. Early and mid-term outcomes. Eur J Cardiothorac Surg 2018;53:1055-61.
Cite this article as: Vojacek J, Zacek P, Ondrasek J. Multiple valve endocarditis: a Hemi-Commando procedure. Ann Cardiothorac Surg 2019;8(6):705-707. doi: 10.21037/ acs.2019.07.07 